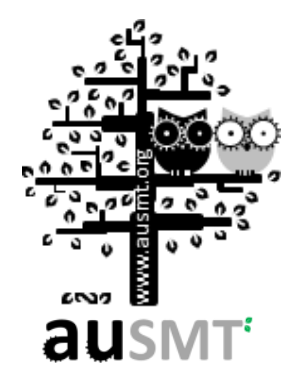

\title{
The Industrial Specialization and Economic Contribution of the Smartphone Supply Chain: The Case of Apple and HTC
}

\section{Wen-Nan Tsan, Chi Chang, Bo-Chi Lin, Wen-Yao Han, and Nan-Shiun Chu*}

\author{
Market Intelligence \& Consulting Institute (MIC), Institute for Information Industry (III), Taiwan \\ (Received 3 August 2012; Published on line 1 September 2012) \\ *Corresponding author: Julian_Chu@micmail.iii.org.tw \\ DOI: $\underline{10.5875 / a u s m t . v 2 i 3.155}$
}

\begin{abstract}
This study examines the linkage between industrial specialization and economic contribution in the smartphone component industry. The value created from the manufacturing process may shift the leading positions of countries involved in the supply chain. In turn, the regional hierarchy will further influence the slicing up of the smartphone component value chain based on each country's comparative advantages. The economic contribution from the Apple and HTC supply chains are analyzed in terms of value added, jobs created and employee composition in Taiwan and other countries. Analysis found that for every smartphone produced, Apple's component supply chain created 37.3 USD of value in Taiwan, as opposed to 58.7 USD for HTC. This study recommends that industrial efforts should further focus on the production of application processors, LCD panels and passive components.
\end{abstract}

Keywords: Industrial specialization; Added value; Smartphone component; Supply chain; Taiwan

\section{Introduction}

\subsection{Background}

In recent years, industry added value has superseded industry output as the preferred indicator for measuring an industry's contribution to national economic growth, as industry output alone does not provide a picture of value composition. Added value, on the other hand, can show how much value a business can create from raw materials to finished goods. From the viewpoint of nation economies, if upstream suppliers are all local firms and all raw materials are supplied domestically, output and added value can stand at parity. Most of the suppliers in the global smartphone components industry are, however, Japanese. The value created in each country after the purchase of raw materials makes a real contribution to its national economy. Thus, given the growth of the smartphone market, a country can create more economic benefits for itself by emphasizing high added-value industries.

This paper conducts a case study through an analysis of data collected from the component supply chain of Apple and HTC, the top selling smartphone vendors worldwide. The development and commercialization of smart handheld devices and advances in wireless communication technology have created explosive growth in the smartphone industry. The industry's component supply chain actively involves many firms, and this paper aims to provide guidance for countries and firms to thrive within this environment. 
The paper proceeds as follows. We begin by reviewing relevant literature on industrial specialization, the Flying Geese Paradigm and the Job Estimation Method, proposing a conceptual framework for this research. Section 2 discusses the smartphone industry and its technological characteristics to provide a better understanding of the industry's dynamics. Section 3 compares and analyzes the industrial specialization of the US, South Korea, Taiwan, Japan, and China in the smartphone component supply chain, and compares the types of jobs created by smartphone industry. Finally, the contribution to the Taiwan economy from Taiwanese smartphone component industry is analyzed and suggestions are proposed for improvements.

\subsection{Smartphone Industry Overview}

In July 2008, Apple released the iPhone 3G, the iPhone to support $3 \mathrm{G}$ service, marking a turning point for the global smartphone industry. The iPhone 36 not only

Wen-Nan Tsan holds a Ph.D. degree in Information Management from Taiwan's National Central University. He is in charge of various projects as project manager, such as ITIS (Industry \& Technology Intelligence Services) program for professional training and research project for intellectual capital environment establishment and promotion and international cooperation. Dr. Tsan received many awards including Annual Outstanding Youth Achievement Award (1995) and MOEA (Ministry of Economic Affairs) Minister's Outstanding Achievement Award (1998). Dr. Tsan has been responsible for numerous projects in MIC, focusing on intellectual capital and information capability enhancement in Taiwan.

Chi Chang received a MBA degree from the National Central University. He is currently a Deputy Director of Market Intelligence \& Consulting Institute at the Institute for Information Industry (III) in Taiwan. Mr. Chang is also the consultant specialized in WiMAX, FTTX, VolP, Broadband, and Mobile communications. He has been in charge of the "M-Taiwan Program", "Telecom Applications Platform Development and Promotion Program" Since 2004 as a sub-project manager. His research work has appeared in journals such as Behaviour \& Information Technology.

Bo-Chi Lin earned his Ph.D. degree from National Taiwan University, focusing on marketing management and consumer behaviors. He is an expert on mobile communications industry research, with research scopes covering the Taiwanese Smartphone Industry research, the development trends of Smartphone products and key components, of mobile phone brand-name vendors and mobile phone operating system developers, and of mobile phone service applications and more. His research work has appeared in journals such as Journal of Business \& Psychology, and Marketing Letters.

Wen-Yao Han holds a Bachelor's degree in Philosophy from Fu Jen Catholic University. He specializes in mobile communications industry research, with areas covering Taiwan Smartphone industry statistics and Smartphone product and key component development. He is in charge of component shipment research of smart handheld device projects.

Nan-Shiun Chu received MBA degree from The City University of New York, Baruch College. He is currently a Senior Industry Analyst \& Senior Manager of Market Intelligence \& Consulting Institute at the Institute for Information Industry (III) in Taiwan. Mr. Chu has joined the "Telecom Applications Platform Development and Promotion Program" Since 2007 and "DTV Value-Added Services Development and Promotion Program" since 2009, as a project team member and sub-project manager respectively. His research work has appeared in journals such as Behaviour \& Information Technology, International Journal of Digital Television, and Journal of Computing. featured a 3.5-inch touchscreen but also launched a proprietary software boutique, the App Store, to support the product. The launch of the App Store was significant in that indicated that the focus of smartphone hardware spec development was shifting to the user's operating experience, with features such as multi-touch and touch gesture. At the same time, the focus of software application development shifted to providing diversified functions catering to consumers' needs, such as A/V entertainment and social networking. With the introduction of iPhone 3G, the market focus for smartphones shifted from business customers to include general consumers.

At the end of 2008, Google unveiled the Android OS platform and the Android Market application store. Similar to Apple's iPhone operating system (iOS), Android also features integration between HMI (Human Machine Interface) and software applications. With its open and highly flexible design, Android has driven down smartphone prices. While Apple succeeded in re-orienting the smartphone market from business to consumer, the introduction of Android has allowed smartphones to further expand their market presence, eroding the market share of feature phones, and gradually dominate the mobile phone market.

Led by Apple and Google, the shipment volume share of smartphones reached $29.0 \%$ of all mobile phone shipments in 2011 , up from $12.2 \%$ in 2008 . Looking ahead, it is expected that hardware component costs will continue to drop, pushing down smartphone prices in the end market as a result. Moreover, with the continuing expansion of $3 G$ services, application development is projected to continue to advance. Smartphone shipment volumes are expected to exceed 1.2 billion units in 2015, growing at a CAGR (Compound Annual Growth Rate) of 33.7\% from 2010 to 2015, accounting for more than $60 \%$ of total shipment volume.

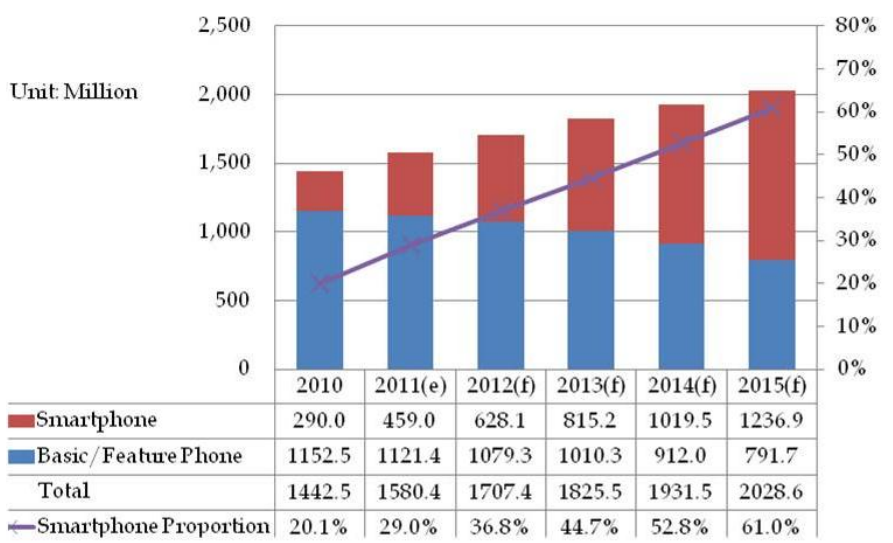

Figure 1. Worldwide Mobile Phone Shipment Volume, 2010 - 2015. 
The choice of operating system plays an important role in the market share of all major branded smartphone vendors. Android's major supporters (HTC and Samsung) are expected to continue to grow their market share. Given the relative maturity of software application ecosystem centered on Apple's iOS and the company's innovative hardware specs, Apple is expected to secure its dominance in the smartphone market. If Meanwhile, Nokia is hoping its Windows 7-based smartphone can replace Symbian in the entry-level smartphone market, thus helping to arrest and reverse its declining market share. Other entry-level smartphone vendors, such as Huawei and ZTE, have cost advantages that mainstream branded vendors lack. The emergence of the entry-level smartphone market is expanding the smartphone market as a whole, giving these firms room to thrive.

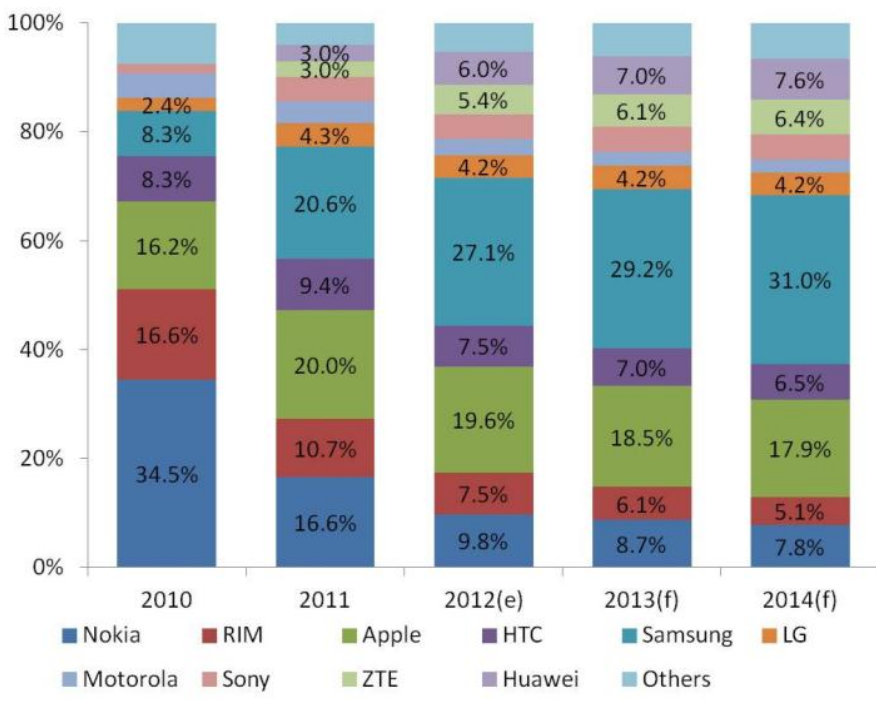

Figure 2. Worldwide Smartphone Vendor Market Share, 2010 - 2014.

Table 1. Global Major Smartphone Branded Vendors' Advantages and Disadvantages.

\begin{tabular}{|c|c|c|c|}
\hline & Advantages & Disadvantages & Market Share Growth Driver \\
\hline Nokia & $\begin{array}{l}\text { High share of emerging markets, } \\
\text { strong supply chain management, } \\
\text { sufficient experience in software } \\
\text { application services }\end{array}$ & $\begin{array}{l}\text { Currently undergoing OS shift: the } \\
\text { first WP7 model will not be } \\
\text { released till year-end and the } \\
\text { market popularity of WP7 } \\
\text { Smartphone remains to be seen }\end{array}$ & $\begin{array}{l}\text { Expanding WP7 platform to } \\
\text { entry-level Smartphones, } \\
\text { combining Nokia services, and } \\
\text { replacing Symbian }\end{array}$ \\
\hline RIM & $\begin{array}{l}\text { Good integration on enterprise } \\
\text { application services }\end{array}$ & $\begin{array}{l}\text { Less appealing to general } \\
\text { consumers }\end{array}$ & $\begin{array}{l}\text { Strengthening consumer } \\
\text { application development }\end{array}$ \\
\hline Apple & $\begin{array}{l}\text { Mature software application } \\
\text { ecosystem, equipped with } \\
\text { innovative HMI design capabilities }\end{array}$ & $\begin{array}{l}\text { Launching merely one model } \\
\text { annually, less likely to attract users } \\
\text { from all market segments }\end{array}$ & $\begin{array}{l}\text { Expanding iPhone series to } \\
\text { entry-level Smartphone market }\end{array}$ \\
\hline Samsung & $\begin{array}{l}\text { Complete component supply within } \\
\text { the group and high technology } \\
\text { capabilities in terms of application } \\
\text { processors, panels and memory }\end{array}$ & $\begin{array}{l}\text { Product design is similar to Apple, } \\
\text { lack of innovation }\end{array}$ & $\begin{array}{l}\text { Leveraging its significant production } \\
\text { scale to join the entry-level } \\
\text { Smartphone market }\end{array}$ \\
\hline HTC & $\begin{array}{l}\text { Long-term platform introduction, } \\
\text { extensive mechanical design } \\
\text { experience, and close relationships } \\
\text { with OS integrators and telecom } \\
\text { operators }\end{array}$ & $\begin{array}{l}\text { Lack of key component design, } \\
\text { relatively low sales volume, } \\
\text { difficulty reducing manufacturing } \\
\text { costs, relatively few patents }\end{array}$ & $\begin{array}{l}\text { Strengthening software } \\
\text { applications to enhance } \\
\text { differentiation }\end{array}$ \\
\hline LG & $\begin{array}{l}\text { Complete component supply chain } \\
\text { within the group }\end{array}$ & $\begin{array}{l}\text { A late-comer to the Smartphone } \\
\text { trend }\end{array}$ & $\begin{array}{l}\text { Launching products with advanced } \\
\text { specs, leveraging LG group-wide } \\
\text { supply chain technologies }\end{array}$ \\
\hline Motorola & $\begin{array}{l}\text { Long-term platform introduction } \\
\text { and extensive mechanical design } \\
\text { experience }\end{array}$ & $\begin{array}{l}\text { Relatively low sales volume, mobile } \\
\text { phone business continues to } \\
\text { operate at a loss, less likely to } \\
\text { invest resources in model } \\
\text { development }\end{array}$ & $\begin{array}{l}\text { Strengthening software } \\
\text { applications to enhance } \\
\text { differentiation }\end{array}$ \\
\hline $\begin{array}{l}\text { Sony } \\
\text { Ericsson }\end{array}$ & $\begin{array}{l}\text { Parent company Sony offers } \mathrm{A} / \mathrm{V} \\
\text { entertainment services, } \\
\text { strengthening product application } \\
\text { development }\end{array}$ & $\begin{array}{l}\text { Relatively low sales volume, mobile } \\
\text { phone business continues to } \\
\text { operate at a loss, less likely to } \\
\text { invest resources in model } \\
\text { development }\end{array}$ & $\begin{array}{l}\text { Integrating } A / V \text { entertainment } \\
\text { services within the group to } \\
\text { enhance user experience }\end{array}$ \\
\hline
\end{tabular}


From the standpoint of smartphone application structure, Apple and HTC have been highly successful in integrating their OS platform and firmware, facilitating the ensuing development of software application systems, and thus are projected to maintain or expand their current market share. However, Samsung and LG have a greater advantage from the perspective of component supply from upstream manufacturers. Currently, Nokia still has the largest market share of any mobile phone vendor, but the firm has shifted its OS strategy, introducing new platform and rebooting product development. While Nokia is dealing with these distractions, its competitors have made rapid advances.

At its initial release, the hardware specs of the iPhone 3G was met with critical comparisons to competing models (e.g., its relatively low-resolution 2-megapixel camera lens). However, Apple designs its products based on a "subtraction" principle, focusing mainly on functions that are most important to users, such as its slim product design and highly-sensitive, vivid touchscreen. Unlike its competitors, which tend to emphasize on integrating all functions, Apple will "subtract" any design that contradicts this principle.

To compete in the smartphone market requires extensive access to the various required component technologies. A smartphone is composed of numerous sophisticated components, including the baseband chipset, application processor, panel, touchscreen, sensor and case. To optimize product design, a vendor must have intimate access to all component technologies. For example HTC not only excels in mechanical design but also has the R\&D resources needed to create all the above-mentioned components. HTC's technology capabilities are even comparable to those of chipset designers and panel manufacturers, enabling the company to produce popular models such as the Diamond, Hero and Desire which all featuring higher grade technology than found in other smartphones.

In addition to component technology, a company has to secure a supply of innovative components for its new products. The current strong market demand for smartphones has resulted in supply shortages of some emerging upstream components, such as AMOLED (Active Matrix Organic Light Emitting Diode) panels. Vertically integrating their supply chains - e.g., by investing in key component companies - gives companies a key competitive advantage.

\subsection{Key Smartphone Component Suppliers}

\section{Application Processors}

Qualcomm leads the application processor market, followed by Texas Instruments and Samsung. Future application processors will be characterized by high clock speeds of $1.5 \mathrm{GHz}-2 \mathrm{GHz}$ and multi-core architecture. Qualcomm is planning to launch a four-core processor, featuring a $28 \mathrm{~nm}$ manufacturing process and a $2.5 \mathrm{GHz}$ clock speed in 2012. In addition, ARM's forthcoming Cortex-A15 processor and Intel's forthcoming Medfield processor are both expected to feature high-performance processing.

The Nvidia Terga 3 ("Kal-El") application processor features a quad-core architecture, $40 \mathrm{~nm}$ process technology and a $1.5 \mathrm{GHz}$ clock speed, which hit the market at the end of 2011. Despite running four Cortex A9 high-performance CPU cores, the Kal-El has a fifth low-leakage CPU core running at operating frequencies below $500 \mathrm{MHz}$, and is designed mainly to handle less demanding processing tasks including web browsing and email. In such circumstances, power consumption will be significantly reduced by using the fifth CPU core alone rather than the four main CPU cores.

In addition, leading brands have begun consolidating a GPU (Graphic Processing Unit) into their processors, typically supporting 1080p full HD output and 3D graphics, thereby helping meet user demand for high-end display applications. While processors are growing faster, leading brands are also working on reducing power consumption. Meanwhile, ARM's new Mali GPU is expected to be widely popular, with the Mali T604 seen as having the potential to significantly improving mobile processing capabilities for gesture control, videoconferencing, and HTML5.

\section{Baseband ICs}

Qualcomm again dominates in the baseband IC market, followed by Mediatek and Texas Instruments. LTE technology is expected to emerge the standard protocol for global 4G communications, and baseband IC suppliers are largely focused on LTE communications R\&D. Qualcomm has the industry's largest portfolio of LTE-related patents, and is continuing to devote significant resources in this area, which is expected to give the company a significant advantage in the competition for the next generation of communications technology. To combat Qualcomm dominance in the $3 \mathrm{G}$ era, Japanese companies such as NTT DoCoMo, NEC, Fujitsu, and Panasonic are expected to collaborate with Samsung in jointly developing broadband ICs. Samsung and LG have also invested heavily in the development of LTE PHY (Physical Layer) technology, jointly holding one-fifth of relevant patents.

\section{Integrated Application Processors and Baseband IC Solutions}

Qualcomm's integrated application processor and baseband IC solutions accounting for over $40 \%$ of the global integrated solution market in 2010. Nvidia 
indicated its intention to launch integrated solutions with its May 2011 acquisition of baseband IC supplier Icera. This has prompted mobile phone IC design houses to begin shifting their development directions towards integrated application processor and baseband IC solutions.

Due to communications patents and technology limitations, Taiwanese manufacturers have produced application processors and baseband ICs based on $2 \mathrm{G}$ communications technology, mainly for use by feature phone vendors and Chinese makers of counterfeit smartphones. To gain access to baseband ICs, in 2009 Taiwanese IC design houses Mediatek entered into a WCDMA (Wideband Code Division Multiple Access) patent agreement with Qualcomm. The agreement exempts Mediatek from paying upfront fees and royalty fees, but Mediatek's customers do not receive rights to any of Qualcomm's patents and technologies.

This agreement shifts the royalty fees to Mediatek's clients but while allowing Mediatek to compete in the global 3G market. In February 2011, Mediatek launched the MT6573, an integrated application processor and baseband IC solution running on ARM 11 architecture and featuring $650 \mathrm{MHz}$ clock speed and HSPA (High Speed Packet Access) technology. This solution has since been integrated into the Lenovo Android-based A60 smartphone, officially launching Mediatek's entry into the $3 G$ era and the smartphone supply chain. The Lenovo A60 smartphone began mass production in August 2011 and sold well, prompting Mediatek to accelerate the launch date of its MT6575 solution, which debuted in the first quarter of 2012.

Currently, Taiwanese baseband IC design houses capable of breaking into the global smartphone supply chain include Mediatek, which supplies WCDMA baseband ICs, and VIA Telecom, which both supply CDMA baseband ICs. Samsung, determined to reduce its dependence on Qualcomm baseband ICs, selected VIA Telecom to supply CDMA baseband ICs for the Samsung Droid Charge, marking a significant victory for a Taiwanese baseband IC design house joining the supply chain of an international brand.

\section{Bluetooth/Wi-Fi/GPS}

Broadcom, Mediatek and Texas Instruments are the major suppliers of integrated Bluetooth/Wi-Fi (Wireless-Fidelity)/GPS (Global Positioning System)/FM ICs, with Broadcom holding a leading position for smartphones.

Broadcom pioneered the development of wireless communications integrated ICs. Its BCM4330 solution supporting Bluetooth 4.0 and Wi-Fi Direct while reducing space and power requirements during the design process

In July 2011 Mediatek launched a SoC (System on
Chip) solution, dubbed the MT 6620, which consolidates Bluetooth 4.0+HS/Wi-Fi $802.11 \mathrm{n} 2.4 \mathrm{GHz}$ and $5 \mathrm{GHz}$ dual-band/GPS/FM, making Mediatek the world's second supplier of four-in-one integrated ICs after Broadcom.

\section{NAND Flash Memory}

Samsung dominates the NAND flash memory market, followed by Toshiba and Hynix. The iPhone 4 and i900 high-end smartphones were launched in 16GB and $32 \mathrm{~GB}$ of flash memory, and strong sales of high-end smartphones doubled demand for NAND flash memory between 2010 and 2011. Meanwhile, Toshiba, Intel and Samsung have individually entered into the development of $19 \mathrm{~nm}, 20 \mathrm{~nm}$, and $21 \mathrm{~nm}$ process technologies. Nevertheless, it is anticipated that continued development towards $10 \mathrm{~nm}$ process technology will face difficulties and result in bottlenecks by 2015 . Consequently, all leading IC design houses have begun shifting technology development to different domains.

Toshiba is shifting its focus to 3D NAND flash memory development while Elpida and Sharp are jointly moving towards ReRAM development and Hynix has focused on MRAM. ReRAM and MRAM ICs are due to hit the market in 2013 and 2014, respectively. Given its ample funds and resources, Samsung is currently developing all three of these flash memory technologies simultaneously, aiming to maintain its leadership in the memory IC market.

Taiwan suffers from inadequate proprietary research and development capacity for mobile phone memory ICs, thus Taiwanese IC design houses mainly supply the entry-level to mid-range mobile phone markets. Powerchip, Taiwan's first NAND flash memory manufacturer, has recently begun to reduce PC DRAM production. Leveraging NAND flash technology licensed from Renesas, Powerchip has begun mass producing $90 \mathrm{~nm}$ and $70 \mathrm{~nm}$ NAND flash memory products while pursuing 40nm technology. Macronix, another Taiwanese flash memory maker, mainly produces NOR flash memory, used in Apple Mac and Macbook Air products.

\section{Mobile DRAM}

Samsung dominates the global mobile DRAM market, followed by Hynix and Elpida. In 2010, high-end smartphones mainly used 512MB mobile DRAMs, increasing to $1 \mathrm{~GB}$ in 2011 for several flagship smartphone models such as the HTC EVO 3D, Samsung Galaxy S2 i9100, and Motorola Atrix. Strong smartphone sales doubled DRAM capacity between 2010 and 2011.

The market for process technology is dominated by leading brands including Korea's Samsung and Hynix, Japan's Elpida (acquired by Micron in May 2012) and Toshiba, and Micron and Intel in the US. Elpida's 30nm LPDDR2 mobile DRAM is a particularly powerful solution. 
Nevertheless, anticipated bottlenecks in the data transmission rate of LPDDR2 mobile DRAM is prompting leading DRAM vendors to begin developing their next generation process technologies. Micron is moving towards the development of LPDDR3 mobile DRAMs, while Samsung and Hynix both are focusing on the development of SPMT (Serial Port Memory Technology) and wide $\mathrm{I} / \mathrm{O}$ standards.

Leading Taiwanese mobile DRAM suppliers have to pay patent license fees to international brands and are divided into two teams. The Micron team includes Nanya Technology and Inotera Technology while the Elpida team has Rexchip, Powerchip, Winbond Electronics, and ProMOS Technologies. Nanya Technology of the Micron team is currently sending samples of four $2 \mathrm{~Gb}$ chips in a QDP (Quad Die Package) to several mobile phone brands, aiming to break into their supply chains. Inotera Technology is capable of producing $50 \mathrm{~nm} 2 \mathrm{~Gb}$ mobile DRAMs.

Elpida began producing mobile DRAMs in the third quarter of 2011, and licensed the technology to Rexchip, thus ensuring sufficient production capacity to meet future increased demand. Powerchip has scaled down standard PC DRAM production, focusing on OEM production instead, producing mobile DRAM licensed from Elpida, with Elpida handling sales. In addition, with NAND flash memory technology acquired from Renesas, Powerchip is expected to launch an integrated MCP for use in mobile devices. Winbond Electronics accounts for over $20 \%$ of the mobile DRAM market, making it Taiwan's biggest mobile DRAM provider and the world's largest PSRAM supplier. However, the company has opted not to compete head-to-head with international chipmakers but rather mainly provides LPDDR mobile DRAMs with lowand medium storage capacity.

\section{Small- and Medium-sized Panels}

The global small- and medium-sized panel market is led by Chimei Innolux, followed by SMD (Samsung Mobile Display) and Sharp. Currently, smartphones mainly use small- and medium-sized TFT-LCD (Thin Film Transistor Liquid Crystal Display) with AMOLED (Active Matrix Organic Light Emitting Diode) is emerging as a possible future replacement. SMD produces $97 \%$ of AMOLED displays.

Panel displays have two process technologies, namely a-Si (Amorphous Silicon) and LTPS (Low Temperature Poly-Si), both which can be used in TFT-LCD and AMOLED displays. The development of a-Si requires less time and is less expensive but provides relatively low resolutions making it appropriate for use in entry-level and mid-range mobile phones. Meanwhile, the more brilliant displays typical of smartphones has spurred a high adoption rate for LTPS despite its higher costs. For

www.ausmt.org

Copyright (C) 2012 International Journal of Automation and Smart Technology instance, the IPS (In-Plane Switching) Retina display used in the iPhone 4 uses LTPS technology, as does AMOLEDs. However, Hitachi recently revealed an a-Si-based 4.5-inch IPS LCD display panel running at $1280 * 720$ and supporting up to $329 \mathrm{ppi}$ (pixel per inch), promising high performance at reduced costs.

IPS TFT-LCDs are widely used in high-end smartphones, featuring a 960*640 resolution, fast response speeds and a wide viewing angle. Samsung is moving towards the development of Super PLS, which it claims will sharpen IPS display performance to WXGA $(1366 * 768)$ resolutions and lower costs.

Working without a backlight, AMOLED is thinner than TFT-LCD and is capable of delivering sharper images with increased brightness, improved contrast ratio, faster response time, wider viewing angles, and reduced power consumption despite its relatively low resolution. The Super AMOLED Plus display offers WVGA $(800 * 480)$ level resolution and is expected to become the mainstream display for use in high-end smartphones.

\section{Passive Components}

Japan's Murata Manufacturing Co. leads the global passive components market, followed by SEMCO (Samsung Electro-Mechanics Corporation) of Samsung Group, and Japan's Taiyo Yuden Co.

Passive components can be divided into resistors, inductors and capacitors, with capacitors accounting for the largest share of the global passive component industry by value. MLCC (Multi-layer Ceramic Capacitor) is the current mainstream capacitor, with a $40 \%$ market share by total shipment value. MLCC can be used for mobile phones, notebook PCs, LCD TVs and digital cameras. The number of capacitors for use in a smartphone ranges between 400 and 500 units, as opposed to 100 and 200 units in conventional mobile phones. This increase in the number of capacitors required, combined with market pressures to produce increasingly compact and lightweight devices has prompted the use of MLCC catering large storage capacity in a small footprint. Murata Manufacturing Co. released the world's first and smallest 0402-size $\mathrm{Hi}-\mathrm{Q}$ type MLCC, where $Q$ is the reciprocal loss rate of the dissipation factor.

Recently global financial turmoil and the appreciation of the yen have left Japanese manufacturers reluctant to expand production. However, resumed growth for demand for mobile phones increased demand for passive components by over $15 \%$ in 2011 . To better distribute risks from natural disasters such as earthquakes, Japan's Murata Manufacturing Co. moved an increasing share of production to China in 2010.

The technology gap between SEMCO and its Japanese rivals is narrowing and SEMCO's production 
capacity grew $150 \%$ between 2007 and 2011. SEMCO has begun to compete on price, aiming to boost its market share. Meanwhile, Taiwanese passive components manufacturers are capable of providing entry-level and mid-range standard MLCCs, with Taiwan's Yageo expanding production in China by $30 \%$, in addition to its four MLCC production lines in Taiwan.

\section{Literature Review}

\subsection{The Flying Geese Paradigm}

The flying geese analogy for regional development [1] posits a hierarchy with a dominant country acting as the advanced pivot and followed by other developing countries. In East Asia today, the paradigm reveals a regional integration with increasing interdependence among regionally linked economies. Thus, the industrial sophistication of the regional cluster increases overall as regional economies take advantage of externalities and linkages.

\subsection{The Job Estimation Method}

The Job Estimation method [2] is used here to analyze the number of jobs created by the smartphone supply chain as a whole, including the factory fraction method and revenue fraction method. In the factory fraction method, knowing that IC manufacturer produces an annual output of 240 million flash chips and has 1,200 employees implies a production per employee ratio which can be generalized to other cases or extrapolated to different scales. In the revenue fraction method, the total production cost as a proportion of the IC manufacturers' revenues can be converted into the number of employees required.

\subsection{Industrial specialization}

Theoretical studies examining motivations for international production sharing basically agree that manufacturing processes can be sliced up into distinct phases, and that significant differences may exist in the respective comparative advantages countries bring to a manufacturing supply chain. Labor-intensive phases are be transferred to less developed countries to take advantage of relatively low production costs including wages and transport. [3] Moreover, studies [4] suggest that slicing these processes up into phases could greatly increase the potential volume of international business. A good that is manufactured in one country could be assembled from components produced in other countries. Consequently, the industry involved in the global production of a finished good may be several times the value added in all production steps.

Reduced transaction costs incentivize companies to slice up the value chain and to locate production sub-processes where manufacturing costs are lowest. Therefore, countries may have a comparative advantage in producing key components, but not necessarily in producing finished goods [5].

"Comparative advantage" [6] is the technological superiority of one country over another in manufacturing products. The preferred approach tries to take advantage of a country's strengths and compensate for its weaknesses. This principle supposes constant productivity, as there is only one factor of production and therefore constant costs that leads to full specialization.

This study examines the linkage between industrial specialization and economic contribution in the smartphone component industry. The value created from the manufacturing process may shift the leading positions among the countries involved in the supply chain. In turn, the regional hierarchy will further influence the slicing up of production phases based on the comparative advantage of the different countries involved. The economic contributions of the Apple and HTC supply chains are analyzed in terms of added value, jobs created and employee composition in Taiwan and other countries.

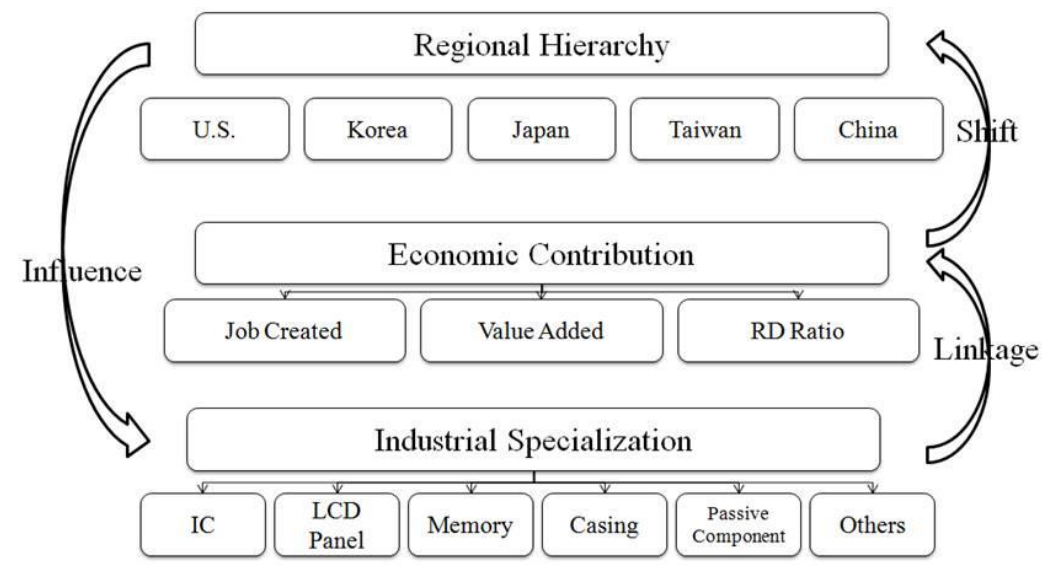

Figure 3. Conceptual Framework. 


\section{Comparison of Industrial}

\section{Specialization in the Smartphone Component Supply Chain}

In 2010, Apple and HTC purchased smartphone components with a total added value of 4.52 billion USD, primarily from the US (1.44 billion USD), Korea (1.39 billion USD), Taiwan (1.12 billion USD), Japan (520 million USD) and China (500 million USD).

\subsection{US}

Leveraging the US's strength in IC design, Apple purchased all of its semiconductor components from the US with the exception of baseband chips (supplied by Infineon in Germany) and memory (supplied by Samsung in Korea). Meanwhile, Qualcomm continued its longstanding partnership with HTC, provided most of the firm's semiconductor components with a complete processor and baseband chip solution.

\subsection{Korea}

Apple only sources memory and a portion of its LCD panels from Korea, while HTC sources a portion of its memory, LCD panels and battery modules there. The high value-added nature of these components (primarily supplied by Samsung or LG ) results in Korea accounting for the highest share of added value.

\subsection{Taiwan}

In the smartphone supply chain, Taiwanese suppliers have leveraged their capacity for mass production to mainly supply medium- and low-value-added standardized products. At the high-value-added end, Taiwanese firms have had some success with LCD panels. Taiwan is also a major supplier of touch screens and casings and is a major supplier of passive components to Apple and HTC.

\subsection{Japan}

Japan's component supply chain is similar to Taiwan's. However, Japan possesses more advanced technology for passive components, printed circuit boards (PCBs) and quartz components. High-end TCXO quart components for example, are priced 10 times higher than lower-grade products. It's worth noting that Japan's share of the component supply chain is lower than Taiwan, but it has control over sources of critical materials and core technologies, including the dielectric ceramic powder used in passive component MLCCs (Multi-layer Ceramic Capacitor), the BT (Bismaleimide-Triazine) resin used in IC substrates and the ceramic base for quartz components. Japan is also a major supplier of LCD panels to Apple and HTC.

\subsection{China}

As the late comer to the smartphone component industry, China only supplies low added value acoustic components and battery modules to Apple and HTC. For example, acoustic component supplier AAC Tech's strength lies in its wide range of product combinations, complete solutions, lower pricing, and customization expertise. Meanwhile, battery module maker ATL is a fully owned subsidiary of Japan's TDK, possessing technical and cost advantages.

Table 2. Distribution of Key Suppliers of Smartphone Components.

\begin{tabular}{|c|c|c|c|c|c|c|}
\hline Added Value & Component & US & Korea & Taiwan & Japan & China \\
\hline \multirow{3}{*}{ Over 10 USD } & Processor & (0)(0) & & & & \\
\hline & Memory & (2) & () () () & & & \\
\hline & Baseband Chip & ()(2) () & & & & \\
\hline \multirow{4}{*}{$6 \sim 10$ USD } & LCD Panel & & (2)(2) & (0) & (2) & \\
\hline & Casing & & & (○) () & & \\
\hline & Passive Component* & & & (0) () & ()() & \\
\hline & Touchscreen & & & (2) (2) () & (2) & \\
\hline $3 \sim 6$ USD & Bluetooth/Wi-Fi/GPS Chip & (2) () () & & & & \\
\hline \multirow{3}{*}{$2 \sim 3$ USD } & PCB/FPC* & (2)(2) & (2) & (2)(2) & (2)(2) & \\
\hline & Camera Module* & & (a) & (2) (2) () & (2) & \\
\hline & Camera Len & & & (2) (2) (2) & & \\
\hline \multirow{8}{*}{ Less than 2 USD } & LED* & & & (2)(2) & (2) () & \\
\hline & Keypad & & & ()(2) () & & \\
\hline & Battery Module* & & (2) & () () & (a) & (a) \\
\hline & \begin{tabular}{|l} 
Amplifier \\
\end{tabular} & ()(2) () & & & (a) & \\
\hline & Acoustic Component* & & & () & & (0)(-) \\
\hline & Touch Control IC* & (0)(-) & & (2) & & \\
\hline & Quartz Component* & & & (2) & (2)(0) & \\
\hline & Connector & & & (2) () () & & \\
\hline
\end{tabular}

Note 1: ()(-) () indicates major supplier, () () indicates secondary supplier and (a) indicates minor supplier. Note 2: Data for components marked with* provided by Fuji Chimera Research Institute. 


\section{Comparison of Manufacturing Locations in the Smartphone Component Supply Chain}

A comparison of MIC* (Territorialism) and MBC *(Nationalism) allows for a deeper analysis of the industrial composition of each country. For example, from a MIC perspective, approximately 79.9 USD of value for Apple's iPhone 4 was produced in Korea. From a MBC perspective, the value created in Korea is approximately 73.8 USD, making Korea the top supplier in both cases (see Table 3).

For the HTC Desire, both MIC and MBC were focused in Taiwan. Notably, the value created by Taiwanese component suppliers for HTC (58.7 USD) was much higher than the value created for Apple (37.3 USD). This demonstrates HTC's contribution to Taiwan's economy as the largest homegrown smartphone brand and its proactive use of local components.

Using MIC and $\mathrm{MBC}$, the difference of value created can be sorted into three categories: $\mathrm{MBC}>\mathrm{MIC}$ US and Japan; MBC = MIC - Taiwan and Korea; MBC < MIC - China. The clearest example is the US and Japan, where $M B C$ values are higher due to their respective comparative advantages [7] as lower-value-added manufacturing operations are moved overseas. As a result, China's lower labor and land costs attract manufacturing industries that rely on cheap labor. While
$\mathrm{MBC}$ and MIC were roughly equal in both Taiwan and Korea, their component industries differed in terms of structure and composition.

\subsection{Korea}

Korea enjoys a particularly strong advantage in the high value-added memory and LCD panel industries and most smartphone components are manufactured locally. Given the high value-added in semiconductor manufacturing, Korea has retained the entire supply chain locally and also manufactures Apple's processor in domestic factories. As a result, the value created by Korea's semiconductor industry is approximately the same from a MIC or MBC perspective.

\subsection{Taiwan}

Taiwanese industries receive smartphone component contracts locally but the locations of manufacturing differ by their added value. High added value semiconductors such as processors, baseband chips, Bluetooth/Wi-Fi/GPS chips, touch control ICs and amplifiers mostly are manufactured in domestic factories while the medium and low added value industries have shifted their manufacturing operations to China or other Asian countries to take advantage of lower labor and production costs.

Table 3. Comparison of Value Created by Each Country per Smartphone

\begin{tabular}{|l|l|l|l|l|l|l|l|}
\hline \multicolumn{2}{|c|}{ Apple iPhone 4 } & Made by & USD & Made in & USD & Made by & USD \\
\hline Made in & USD & Korea & 73.81 & Taiwan & 60.87 & Taiwan & 58.68 \\
\hline Korea & 79.92 & Taiwan & 37.33 & China & 57.85 & US & 51.61 \\
\hline China & 44.87 & US & 29.47 & Korea & 24.67 & Japan & 31.59 \\
\hline Taiwan & 33.91 & Japan & 17.80 & Japan & 19.48 & South Korea & 25.72 \\
\hline Japan & 9.02 & China & 2.96 & US & Other & 4.40 & Other \\
\hline Other & 8.06 & Other & 22.31 & 3.13 & 2.18 \\
\hline
\end{tabular}

Note: The concept of "Made by" refers to the nationality of the firm that receives the customer order, while "Made in" refers to the country where the factory is located (regardless of whether it is owned by the contract manufacturer or the work is outsourced from the contract manufacturer). 


\section{Comparison of Jobs Created by Smartphone Industry}

Based on the Job Estimation method, this study found that the Apple iPhone series has created 65,928 jobs for Apple and its suppliers, compared to 39,519 jobs for HTC. If assembly is also taken into account calculation, the overall value chain for HTC accounts for 17,498 jobs, far higher than the 6,422 jobs created by Apple.

Furthermore, in addition to jobs created, the RD ratio is another indicator used for measuring the added value of these countries. Figures 4 and 5 below show the RD proportions of total employees in Apple's iPhone business and HTC's smartphone business. In both cases, China's RD ratio accounted for less than $1 \%$ of all employees while the US had the highest RD ratio of over $40 \% \quad(43.3 \%$ for the iPhone vs. $67.9 \%$ for HTC smartphones). Taiwan, Japan and Korea all had around the same RD ratio in the low $20 \%$. If Taiwan wants to develop as a technology powerhouse, this ratio of RD personnel needs to be increased.

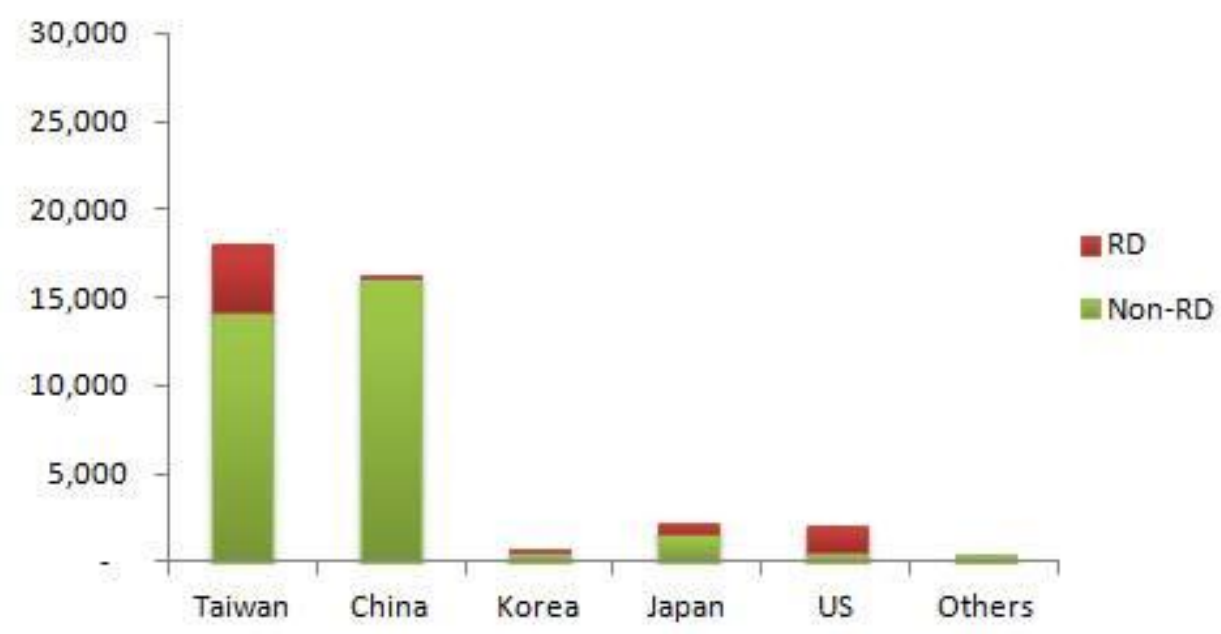

Figure 4. Job Creation in Global Network: The Case of Apple iPhone. (Note: Based on the number of jobs created in 2010 when Apple shipped a total of 47 million smartphones.)

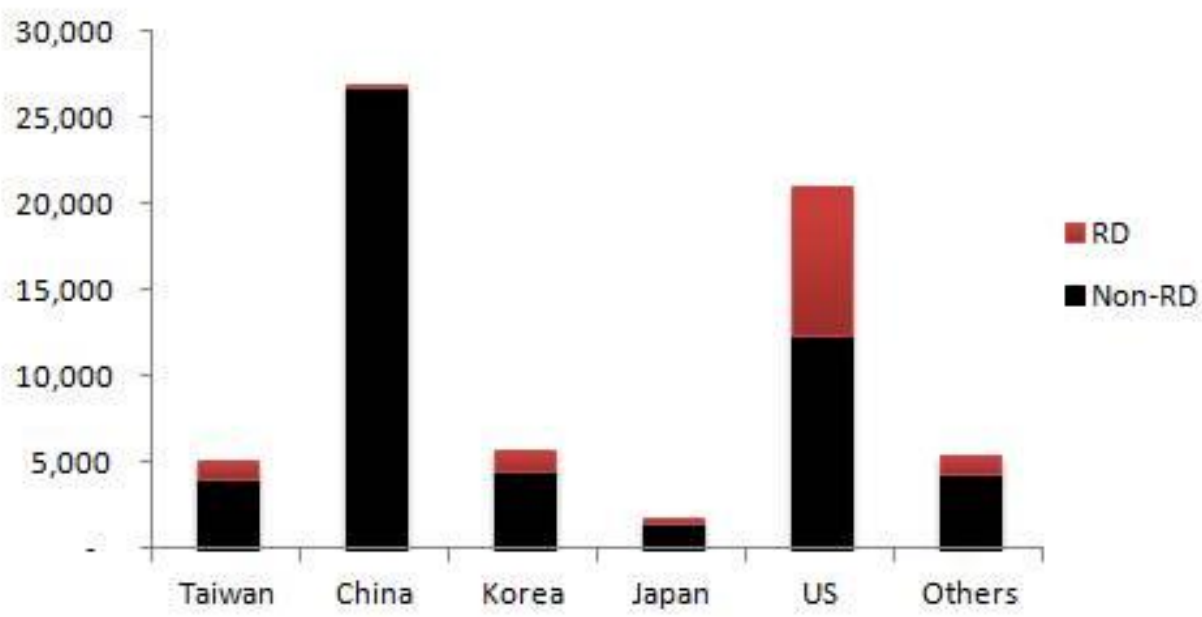

Figure 5. Job Creation in Global Network: The Case of HTC. (Note: Based on the number of jobs created in 2010 when HTC shipped a total of 25 million smartphones.) 


\section{Contribution to the Taiwan Economy by the Taiwanese Smartphone Components Industry}

This study used three dimensions to examine contributions by the Taiwanese components industry: the number of employees, RD employee ratio and added value (see Table 4). The effect on the economy was analyzed to assess the relative contributions of the various component industries. When evaluating how many jobs in Taiwan can be created under the assumption of a $10 \%$ share increase in Apple and HTC component orders, the result will pinpoint which development areas and components should be the focus of future efforts. For example, if Apple and HTC increase their purchases for LCD panels or casings by $10 \%$, Taiwan will gain 185 jobs for LCD panels, as opposed to 72 jobs for casings (based on 2010 shipments).

In Taiwan, the industries that have high added value and create more local jobs include LCD panels, touchscreens, passive components, processors, casings, Bluetooth/Wi-Fi/GPS chips and memory design. Casings, Bluetooth/Wi-Fi/GPS chips and PCB/FPC manufacturing make a greater contribution to the Taiwan economy compared to other component manufacturers. However, inadequate technological competence in baseband, processor, and memory remain a serious issue in Taiwan's smartphone supply chain.

Table 4. Comparison of Economic Contribution from Apple and HTC's Taiwan Smartphone Component Suppliers.

\begin{tabular}{|c|c|c|c|c|}
\hline Component & $\begin{array}{l}\text { Jobs Created in } \\
\text { Taiwan }\end{array}$ & RD Employee Ratio & Value Added & $\begin{array}{l}\text { Jobs Created from } \\
10 \% \text { Contract Share } \\
\text { Increased }\end{array}$ \\
\hline Baseband Chip Manufacturing & Moderate to High & Moderate to High & Moderate & 102 \\
\hline $\begin{array}{l}\text { Bluetooth/Wi-Fi/GPS Chip } \\
\text { Manufacturing }\end{array}$ & Moderate & Moderate to High & Moderate to High & 67 \\
\hline Touchscreen & High & Moderate & Moderate to High & 126 \\
\hline Passive Components & High & Low & Moderate to High & 529 \\
\hline Processor & Low & Moderate to High & Moderate to High & 76 \\
\hline Camera Lens & High & Low & Moderate & 152 \\
\hline PCB/FPC & Moderate to High & Low & Low & 339 \\
\hline Casing & Moderate & Low & High & 72 \\
\hline LCD Panel & Low & Moderate to High & High & 185 \\
\hline Touch Control IC Manufacturing & Low & Moderate to High & Low & 20 \\
\hline LED & Low & Low & Low & 101 \\
\hline Amplifier Manufacturing & Low & Low & Low & 56 \\
\hline Connector & Low & Low & Low & 32 \\
\hline Camera Module & Low & High & Low & 27 \\
\hline Acoustic Components & Low & Moderate to High & Low & 36 \\
\hline Battery Module & Low & Moderate to High & Low & 24 \\
\hline Quartz Components & Low & Low & Low & 56 \\
\hline Touch Control IC Design & Low & High & Low & 24 \\
\hline Buttons & Low & Low & Low & 10 \\
\hline Baseband Chip/Processor Design* & NA & High & High & 315 \\
\hline Memory* & NA & Moderate to High & High & 216 \\
\hline
\end{tabular}

Note 1: For jobs created in Taiwan, Very High means over 1,101 people, High means 751 1,100 people, Medium means $401 \sim 750$ people and Low means under 400 people.

Note 2: For RD Employee Ratio, Very High means over 45\%, High means $31 \sim 45 \%$, Medium means $16 \sim 30 \%$ and Low means under $15 \%$.

Note 3: For value added, Very High means over 5 USD, High means $3.5 \sim 5$ USD, Medium means $2.1 \sim 3.4$ USD and Low means under 2 USD. 


\section{Conclusion}

Currently, Taiwan's component suppliers mainly support entry-level and mid-range smartphones in emerging markets, and technology limitations and patent issues pose significant obstacles for Taiwanese application processor, baseband IC, mobile DRAM, and NAND flash memory manufacturers to become component suppliers for high-end product offerings for first-tier smartphone vendors. Taiwan's competitiveness in manufacturing components for use in entry-level and mid-range smartphone has given Taiwanese manufacturers an advantage by tapping into emerging market demand while differentiating themselves from international leading component suppliers. It is anticipated, however, that these leading suppliers are likely to initiate a price reduction strategy, reducing the price of entry-level components and hindering the development of Taiwanese component suppliers.

The study shows that the industrial specialization of the smartphone component industry in each country reflects the Flying Geese Paradigm [8]. The US is the lead goose with Korea, which has recently supplanted Japan. Taiwan is currently in the middle of the flock and has made significant gains in all component industries except for IC design. China is currently the back of the flock and mainly involved within the manufacturing side. Many industries are, however, gradually transferring their technology to China because of market potential and cost issues. For example, Chinese-made acoustic components are reaching world-class levels and China's rise may bring other countries such as Vietnam into the flock.

In both Taiwan and Korea, the semiconductor industry is the largest smartphone component industry by output. Korea not only manufactures semiconductor products but is also able to secure orders for high added-value memory design. On the other hand, while Taiwan leads the world in wafer foundries, it is relatively weak in IC design.

When looking for component industries that will offer a greater contribution to Taiwan's economic development, this study recommends focusing on the application processor, LCD panel and passive component industries. Nevertheless, a country's comparative advantage in a product can change because determinants of comparative advantage, including technology, specialization, demand characteristic, business practices, and government policies, shift continuously.

\section{References}

[1] S. Kasahara, "The flying geese paradigm: A critical study of its application to east asian regional development," in United Nations Conference on Trade and Development, Geneva, 2004, no. 169. Available: http://unctad.org/en/docs/osgdp20043 en.pdf

[2] G. Linden, J. Dedrick, and K. L. Kraemer, "Innovation and job creation in a global economy: The case of Apple's iPod," Journal of International Commerce and Economics, pp. 223-239, 2009.

Available:

http://www.usitc.gov/journals/08 LindenDedrickKr aemer InnovationJobCreationiPod.pdf

[3] F. Ng and A. Yeats, Production sharing in east asia: Who does what for whom and why? Washington, DC: World Bank Publications, 1999. doi: $10.1596 / 1813-9450-2197$

[4] P. Krugman, R. N. Cooper, and T. N. Srinivasan, "Growing world trade: Causes and consequences," Brookings Papers on Economic Activity, vol. 1995, no. 1, pp. 327-377, 1995.

Available: http://www.jstor.org/stable/2534577

[5] L. Krempel and T. Plümper, "International division of labor and global economic processes: An analysis of the international trade in automobiles," Journal of World-Systems Research, vol. V, no. 3, pp. 487-498, 1999.

Available:

http://jwsr.ucr.edu/archive/vol5/number3/krempel/

[6] P. Kowalski and N. Bottini, "Comparative advantage and export specialisation mobility," in Globalisation, comparative advantage and the changing dynamics of trade, Paris: Organization for Economic Co-operation and Development (OECD) 2011, pp. 81-119. doi: $10.1787 / 9789264113084-5-e n$

[7] A. Mody, "Institutions and dynamic comparative advantage: The electronics industry in south Korea and Taiwan," Cambridge Journal of Economics, vol. 14, no. 3, pp. 291-314, 1990.

Available: http://cje.oxfordjournals.org/content/14/3/291.citation

[8] T. Ozawa, Institutions, industrial upgrading, and economic performance in Japan: The 'flying-geese' paradigm of catch-up growth. Northhampton, Mass.: Edward Elgar Publishing, 2005. 